\title{
Cuidados de enfermagem na depressão pós-parto: estudo na literatura
}

\author{
Nursing care in postpartum depression: a study in literature \\ Atención de enfermería en depresión posparto: un estudio en literatura
}

\begin{abstract}
RESUMO
Objetivo: descrever os achados científicos referentes aos cuidados de enfermagem na depressão pós-parto. Método: Revisão integrativa da literatura, qualitativa e descritiva. Realizado nas bibliotecas da Biblioteca Eletrônica Científica Online e Google acadêmico. Utilizando os descritores: Depressão; Pós-parto; Enfermagem; Cuidados e Saúde, cruzados pelo operador booleano AND, conforme os critérios de inclusão com estudos entre os anos de 2016 a 2021. Foram reunidas 8 publicações referentes a temática e organizadas em um quadro expositivo. Resultados: Predominaram-se artigos dos anos de 2016 e 2020, cada um com 2 artigos publicados. Os demais anos de publicações obtiveram apenas 1 publicação. Os resultados apontaram, de forma geral, que a detecção precoce dos sintomas é um dos cuidados mais prevalentes na enfermagem. Em seguida, observou-se a necessidade da capacitação dos profissionais, para melhor oferecer a sua assistência. Conclusão: Os cuidados de enfermagem são essenciais para a prevenção e tratamento da DPP.
\end{abstract}

DESCRITORES: Depressão; Pós-parto; Enfermagem; Cuidados; Saúde.

\section{ABSTRACT}

Objective: to describe the scientific findings regarding nursing care in postpartum depression. Method: Integrative literature review, qualitative and descriptive. Carried out in the SciELO and Google academic libraries. Using the descriptors: Depression; Post childbirth; Nursing; Care and Health, crossed by the Boolean operator AND, according to the inclusion criteria with studies between the years 2016 to 2021 . Eight publications related to the subject were gathered and organized in an exhibition board. Results: There was a predominance of articles from the years 2016 and 2020 , each with 2 articles published. The other years of publications had only 1 publication. The results showed, in general, that early detection of symptoms is one of the most prevalent care in nursing. Then, there was a need for training professionals to better offer their assistance. Conclusion: Nursing care is essential for the prevention and treatment of PPD.

DESCRIPTORS: Depression; Post childbirth; Nursing; Care; Health.

\section{RESUMEN}

Objetivo: describir los hallazgos científicos sobre el cuidado de enfermería en la depresión posparto. Método: Revisión bibliográfica integradora, cualitativa y descriptiva. Realizado en las bibliotecas académicas SciELO y Google. Utilizando los descriptores: depresión; Post parto; Enfermería; Cuidado y Salud, atravesado por el operador booleano AND, según los criterios de inclusión con estudios entre los años 2016 a 2021. Se reunieron y organizaron ocho publicaciones relacionadas con el tema en un tablero de exhibición. Resultados: Predominó los artículos de los años 2016 y 2020, cada uno con 2 artículos publicados. Los otros años de publicaciones tuvieron solo 1 publicación. Los resultados mostraron, en general, que la detección precoz de los síntomas es uno de los cuidados más prevalentes en enfermería. Entonces, surgió la necesidad de capacitar a los profesionales para ofrecer mejor su asistencia. Conclusión: la atención de enfermería es fundamental para la prevención y el tratamiento de la depresión posparto.

DESCRIPTORES: Depresión; Post parto; Enfermería; Cuidado; Salud.

RECEBIDO EM: 08/06/21 APROVADO EM: 14/06/21

\author{
Kátia Cristina Barbosa Ferreira \\ Enfermeira. Universidade Estadual da Paraíba. \\ ORCID: 0000-0002-3353-2973.
}

\section{Thais Bastos Leite}

Enfermeira. Faculdade de Enfermagem Nova Esperança.

ORCID: 0000-0002-2423-9003. 
Luana Gomes Leitão Rodrigues

Enfermeira. Centro Universitário de João Pessoa.

ORCID: 0000-0001-5259-1357.

\section{Lúcia Gomes de Souza Silva}

Enfermeira. Faculdade Mauricio de Nassau.

ORCID: 0000-0001-6998-435X.

Luciene de Souza Santos Albuquerque

Enfermeira. Centro Universitário de João Pessoa.

ORCID: 0000-0001-5119-195X.

\section{Maria Clara Ribeiro Costa}

Enfermeira. Centro Universitário de João Pessoa.

ORCID: 0000-0003-4422-3719.

\section{INTRODUÇÃO}

A depressão é um fenômeno de altos índices no mundo, atingindo em média 350 milhões de pessoas, o que representa $5 \%$ da população total do mundo, e ainda, é uma das patologias mais prevalentes no Brasil. Continuamente, a depressão está na lista de classificação do Manual Diagnóstico de Transtornos Mentais, descrita como uma patologia gerada pelo Transtorno do Humor ${ }^{(1)}$.

$\mathrm{Na}$ gravidez, existe uma recorrência de casos, por ser um momento de fragilidade da mulher, deixando-a mais suscetível psicologicamente. Diante do nascimento do bebê, e de todos os afazeres que a mulher precisa desempenhar junto com sua família, torna-se uma situação potencial para a Depressão Pós-parto (DPP). É importante diferenciar outras alteraçôes psicológicas que podem ocorrer no pós-parto, como por exemplo o baby blues, que se encerra nos primeiros 15 dias puerperais, ou seja, é um processo transitório, mas que pode ser confundido na identificação dos sintomas da DPP ${ }^{(1-2)}$.

Sequencialmente, a DPP ocorre nas primeiras quatro semanas de puerpério, porém, os sinais do transtorno iniciam na gestação, como visto anteriormente. A DPP é cercada por stress, comportamentos obsessivos, compulsivos, ansiedade e outros aspectos que definem o seu acontecimento. Compreende-se, que o puerpério é um dos momentos que a mulher necessita de apoio familiar e dos profissionais de saúde, sendo primordial, a presença do enfermeiro e de suas ações de prevenção e promoção à saúde da mulher ${ }^{(1-2)}$.

O puerpério apresenta mudanças de grande repercussão na vida da mulher e de sua família, fazendo-se necessário, uma assistência integralizada, pautada na empatia, na troca de saberes, paciência e compartilhamento de ações ${ }^{(3-4)}$. O enfermeiro consegue identificar sintomas que sinalizam a DPP, e principalmente, perceber antes mesmo que a doença se instale, realizando estratégias no lar da gestante ${ }^{(3-4)}$. Sendo assim, construiu-se a seguinte questão de pesquisa: Como se dá os cuidados de enfermagem na depressão pós-parto na literatura atual? Tem-se por objetivo: descrever os achados científicos referentes aos cuidados de enfermagem na depressão pós-parto.

\section{MÉTODO}

Estudo de revisão integrativa da literatura, qualitativo e descritivo. Realizado nas bibliotecas da SciELO (Biblioteca Eletrônica Científica Online) e Google acadêmico. Utilizando os descritores: Depressão; Pós-parto; Enfermagem; Cuidados e Saúde. Estes descritores, foram cruzados pelo operador booleano AND, diante dos seguintes critérios de inclusão: publicações entre os anos de 2016 a 2021, em português, inglês e espanhol, sem duplicidade e que respondessem ao objetivo do estudo. Foram excluídos: publicações fora da linha temporal e que não estivessem de acordo com a proposta da pesquisa. A coleta de dados ocorreu em junho de 2021.

As publicações foram fichadas e organizadas por um instrumento elaborado pelas autoras, no qual foram sintetizadas as seguintes informações: autores, título, ano de publicação, periódico e os cuidados de enfermagem na DPP. A busca inicial permitiu encontrar 250 publicações em geral, após o filtro da linha temporal, apenas 77 publicações seguiram na análise. As 77 publicações tiveram os títulos averiguados, e somente 25 poderiam seguir na pesquisa, diante do que se buscava no estudo. Foram analisados os resumos das 25 publicações, de forma minuciosa. Após a leitura, 10 publicações seguiram na análise. Houve a leitura integral de 10 publicações, porém, apenas 8 publicações foram incluídas nos resultados. Ressalto, que as publicações excluídas, não correspondiam aos critérios de inclusão do estudo, perfazendo 8 publicações em seus resultados. A busca foi validada por dois pesquisadores relacionados à temática.

\section{RESULTADOS}

O quadro abaixo representa a consolidação dos achados científicos nas publicações resultantes da busca e seleção nas bibliotecas eletrônicas.

Predominaram-se artigos dos anos de 2016 e 2020, cada um com 2 artigos publicados. Os demais anos de publicações obtiveram apenas 1 publicação. Os resultados 


\section{QUADRO 1- Apresentação das publicações científicas referentes aos cuidados de enfermagem na DPP:}

\begin{tabular}{|c|c|c|c|c|}
\hline Autores & Título & $\begin{array}{l}\text { Ano de } \\
\text { publicação }\end{array}$ & Periódico & Cuidados de Enfermagem na Depressão Pós-parto \\
\hline $\begin{array}{l}\text { Leonidas FM, } \\
\text { Camboim FEF. }\end{array}$ & $\begin{array}{l}\text { Cuidado de enfermagem à } \\
\text { mulher com depressão } \\
\text { pós-parto na atenção } \\
\text { básica. }\end{array}$ & 2016. & $\begin{array}{l}\text { Temas em } \\
\text { Saúde. }\end{array}$ & $\begin{array}{l}\text { - Diagnóstico precoce da doença. } \\
\text { - Início do tratamento e rápida recuperação da mulher. }\end{array}$ \\
\hline & & & & - Prevenção e detecção \\
\hline $\begin{array}{l}\text { Gomes LAP, Mo- } \\
\text { reira AS da. }{ }^{(4)}\end{array}$ & $\begin{array}{l}\text { Depressão pós-parto: a } \\
\text { visão da enfermagem. }\end{array}$ & 2018. & $\begin{array}{l}\text { Archives } \\
\text { of Health } \\
\text { Investiga- } \\
\text { tion. }\end{array}$ & $\begin{array}{l}\text { - Auxiliar na identificação dos sintomas, } \\
\text { - Ampliação da compreensão da mulher } \\
\text { e do companheiro sobre a patologia. }\end{array}$ \\
\hline Melo GB.(5) & $\begin{array}{l}\text { Assistência de Enfermagem } \\
\text { na prevenção e atenção à } \\
\text { mulher com depressão pós } \\
\text { - parto. }\end{array}$ & 2019. & $\begin{array}{l}\text { Cadernos de } \\
\text { Graduação. }\end{array}$ & $\begin{array}{l}\text { - Implementação de estratégias preventivas para } \\
\text { depressão pós-parto. }\end{array}$ \\
\hline $\begin{array}{l}\text { Silva Braga L. et } \\
\text { al }^{(6)}\end{array}$ & $\begin{array}{l}\text { Assistência de enfermagem } \\
\text { na depressão pós-parto. }\end{array}$ & 2021. & $\begin{array}{l}\text { Estudos } \\
\text { Avançados } \\
\text { sobre Saúde } \\
\text { e Natureza. }\end{array}$ & $\begin{array}{l}\text { - Necessita de mais qualificação na enfermagem. } \\
\text { - Grande parte dos enfermeiros encontram uma barreira } \\
\text { quanto a identificação dos sinais e sintomas das puérpe- } \\
\text { ras com DPP. }\end{array}$ \\
\hline
\end{tabular}

Sousa PHSF. et Enfermagem na prevenção al. ${ }^{(7)}$

Tolentino EC da. et al. ${ }^{(8)}$

Lima SS. et al. ${ }^{(9)}$
Depressão Pós-Parto: Conhecimento sobre os sinais e sintomas em puérperas.

Depressão Pós-Parto: um olhar criterioso da equipe de enfermagem.
Brazilian

2020.

Journal of

Develop-

ment.
- Detecção da Depressão Pós-Parto, devendo esta ocorrer o mais breve possivel.

- A educação em saúde é um fator crucial no desenvolvimento de estratégias no cuidado e tratamento para as puérperas.
2018.
- Detecção dos sinais e sintomas da depressão pós-parto precocemente, com o intuito de impedir o sofrimento das mães e maiores consequências para o bebê.
Revista de Ciências da Saúde Nova Esperança.

\footnotetext{
- Necessidade de capacitação dos profissionais para detectar e prevenir a DPP.
} 


\section{artigo}

Ferreira, K. C. B., Leite, T. B., Rodrigues, L. G. L., Silva, L. G. S.,Albuquerque, L. S. S., Costa, M. C. R.

Cuidados de enfermagem na depressão pós-parto: estudo na literatura

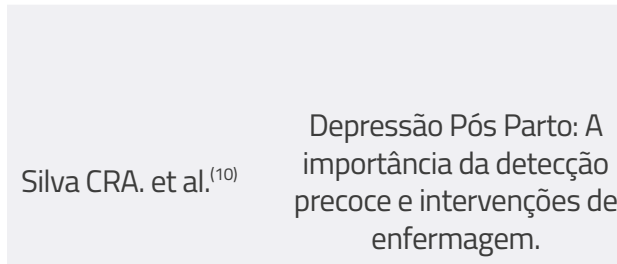

Depressão Pós Parto: A

importância da detecção

enfermagem.

- Minimização de intercorrências no pós-parto, voltadas à a depressão

Revista

Brasileira Interdisciplinar em saúde.
- Orientação familiar

- Educação em saúde para a mãe e família sobre a DPP.

- Detecção precoce.

apontaram, de forma geral, que a detecção precoce dos sintomas é um dos cuidados mais prevalentes na enfermagem. Em seguida, observou-se a necessidade da capacitação dos profissionais, para melhor oferecer a sua assistência.

\section{DISCUSSÃO}

Os estudos expuseram, que existe uma série de sentimentos e emoções em torno da puérpera, e que estes, refletem em diferentes reações e sintomas da DPP em seu dia-a-dia. Um dos resultados visto frequentemente, revelou que os sintomas são identificados pelos enfermeiros, sendo este um dos cuidados essenciais, e que pode auxiliar na prevenção de maiores agravos na DPP. $\mathrm{O}$ enfermeiro consegue identificar em seu cuidado: choro recorrente, irritabilidade, desespero na realização das atividades com o bebê, tristeza persistente e instabilidade emocional e de humor ${ }^{(3-45-6-7-89-910)}$.

É possível destacar, que o enfermeiro realiza orientações, fortalece a família, oferece apoio emocional, conforta e proporciona tranquilidade para a puérpera. $\mathrm{O}$ fato de ajudar a mãe e a família é importante no quesito de suporte terapêutico, antes ou durante o tratamento da DPP. A assistência de enfermagem alcança todo o ciclo gravídico-puerperal da mulher e de sua família, com um olhar singular, formação de vínculo, auxiliando nas necessidades identificadas, medos e insegurançass ${ }^{(3-45-4-6-7-89-10)}$.

O acompanhamento da puérpera com DPP é multiprofissional, ela precisa de todos os profissionais que possam trabalhar com integralidade em seu cuidado. $\mathrm{O}$ enfermeiro reconhece os sintomas relacionados a DPP, e por isso, precisa se capacitar frequentemente, para identificar esse diagnóstico precocemente junto a toda equipe. A continuidade do cuidado acontece no diálogo, nas interconsultas dos profissionais, na compreensão dos sentimentos expressados pelas gestantes e puérperas e principalmente no apoio para enfrentar a situação clínica $^{(3-455-6-7-7 \cdot 9-9-10)}$.

O contexto familiar influencia, por isso, as orientações de enfermagem quanto ao manejo com o bebê e as ações compartilhadas referentes aos cuidados diários do recém-nascido, buscando apoiar a mãe, são importantes. Os cuidados de enfermagem envolvem a troca de informaçóes, esclarecimento de dúvidas, sensibilidade com o outro, empatia e valorização dos sentimentos. As práticas de enfermagem são embasadas cientificamente, utilizando de teorias e técnicas de relacionamento e comunicação interpessoal para uma assistência qualificada (3-45-6-6-7-9-9-10)

Os resultados deste estudo, demonstrou que há necessidade de novos programas relacionados a saúde pública, bem como, no- vas intervenções, para elaborar estratégias que modifiquem os indicadores de DPP, e permitam avançar na área obstétrica ${ }^{(11)}$. A DPP é uma das complicações obstétricas maternas, que demonstram o quão amplo é o processo gravídico-puerperal, e que além disso, é preciso relacionar o acontecimento da DPP com outros fatores, e não somente os hormonais, mas os financeiros, sociais, econômicos e de relações familiares ${ }^{(11)}$.

\section{CONCLUSÃO}

Este estudo alcançou o seu objetivo, trazendo os cuidados de enfermagem na DPP de forma organizada e sistematizada, diante de publicações atuais sobre o tema, fortalecendo a prática profissional da enfermagem e saúde.

Percebeu-se que todos os artigos estudados compartilharam de resultados semelhantes, abordaram a importância do enfermeiro no cuidado continuado, integral, empático e educacional da puérpera e sua família diante da DPP. Ressalto, que os cuidados de enfermagem na DPP começam desde o pré-natal, como foi explanado nos resultados. Recomenda-se este artigo, para todos os interessados em conhecer a temática, especialmente, médicos, enfermeiros, acadêmicos da área da saúde e a equipe multiprofissional que proporciona o cuidado.

\section{REFERÊNCIAS}

1- Félix, TA. et al. Atuação da enfermagem frente à depressão pós-parto nas consultas de puericultura. Enfermería Global. $n^{\circ} 29$, 2013.

2- Organização Pan-Americana de Saúde. Depressão. 2020.

3- Leonidas FM, Camboim FEF. Cuidado de enfermagem à mulher com depressão pós-parto na atenção básica. Temas em Saúde. v.16, n.3, 2016.

4- Gomes LAP, Moreira AS da. Depressão pós-parto: a visão da enfermagem. Archives of Health Investigation. v.7, 2018.

5- Melo GB. Assistência de Enfermagem na Prevenção e Atenção à 


\section{REFERÊNCIAS}

Mulher com Depressão Pós - Parto. v.5, n.2, 2019.

6- Silva Braga L. et al. Assistência de Enfermagem na Depressão Pós-parto. Estudos Avançados da Natureza. v.1, 2021.

7- Sousa PHSF. et al. Enfermagem na prevenção da depressão pós-parto. Brazilian Journal of Development. v.6, n.10, 2020.

8- Tolentino EC da. et al. Depressão Pós-Parto: Conhecimento sobre os sinais e sintomas em puérperas. Revista de Ciências da Saúde Nova Esperança. v.14, n. 1, 2016.
9- Lima SS. et al. Depressão pós-parto: um olhar criterioso da equipe de enfermagem. Cadernos de Graduação. v.4, n.3, 2018.

10- Silva CRA. et al. Depressão Pós Parto: A importância da detecção precoce e intervenções de enfermagem. Revista Brasileira Interdisciplinar em saúde. v.2, n.2, 2020.

11. Santos LJ, Raifur GN, Antunes MB, Almeida CR, Bolsoni LLM, Oliveira WT, Charlo PB. Complicações do pós-parto em mulheres que realizaram o pré-natal no SUS. Revista Saúde Coletiva de Baueri. 2019. 